\title{
Severe interstitial lung disease following treatment with certolizumab pegol: a case report
}

\author{
To the Editor:
}

Over the past decade, the understanding of autoimmune diseases and the development of biological agents have radically changed the treatment paradigm for severe rheumatoid arthritis. Several tumour necrosis factor (TNF)- $\alpha$ inhibitors are available for clinical use, relieving symptoms and reducing the likelihood of relapse in patients not responding successfully to disease-modifying antirheumatic drugs; however, there are limited data regarding the safety of, in particular the newest, TNF- $\alpha$ inhibitor.

Drug-associated interstitial lung disease is a well-known serious and potentially fatal adverse effect of early TNF- $\alpha$ inhibitor use. Being a pegylated humanised Fab monoclonal antibody, certolizumab pegol (CZP) is different from other TNF- $\alpha$ inhibitors. In clinical trials it has been shown to be just as, or even more, efficacious in the treatment of rheumatoid arthritis in combination with methotrexate compared with some of the earlier TNF- $\alpha$ inhibitors [1]. According to the US Food and Drug Administration and the European Medicines Agency, there have been no severe non-infectious adverse lung effects of CZP use. However, a systematic review of the literature revealed two previous cases of CZP-associated interstitial lung disease, one with fatal outcomes $[2,3]$.

This is a report of a patient with rheumatoid arthritis who developed severe acute pneumonitis after treatment with CZP. The patient is a 72-year-old female who had suffered from severe erosive seropositive (rheumatoid factor and anti-cyclic citrullinated peptide) rheumatoid arthritis for 43 years. 1 year earlier she had had a pacemaker fitted after a non-ischaemic cardiac arrest. She was an ex-smoker since 2005 with an accumulated tobacco consumption of $\sim 25$ pack years.

For several years the disease control of her rheumatoid arthritis using different disease-modifying antirheumatic drugs had been limited and she was started on CZP. She was treated with CZP intravenously at 2-week intervals in combination with methotrexate. The first three doses were of $400 \mathrm{mg}$ followed by $200 \mathrm{mg}$ every second week.

After receiving a cumulative dose of $1400 \mathrm{mg}$ she began complaining of a dry cough and shivering and was admitted to an emergency clinic at another hospital. Her husband informed staff that, within the past weeks, she had been incoherent for brief periods, and had complained of shortness of breath and generally enhanced fatigue.

At the time of emergency admission her blood pressure was normal 142/72, her pulse rate was slightly increased to 116 beats per min and her temperature was elevated to $37.7{ }^{\circ} \mathrm{C}$. She was in acute respiratory distress with dyspnoea at speech, a respiratory rate of 24 breaths per min and a saturation of $94 \%$ without oxygen supply. There was widespread bilateral rough rales by auscultation. A blood test showed elevated C-reactive protein $\left(458 \mathrm{mg} \cdot \mathrm{L}^{-1}\right)$, neutrophil leukocytosis $13 \times 10^{9}$ cells $\cdot \mathrm{L}^{-1}$ and hyponatriaemia $129 \mathrm{~mol} \cdot \mathrm{L}^{-1}$; other blood samples were normal. An ECG showed sinus tachycardia and echocardiography was normal. Her pacemaker was tested and no abnormalities were found.

Her CURB65 score (confusion, urea $>7 \mathrm{mmol} \cdot \mathrm{L}^{-1}$, respiratory rate $\geqslant 3$ breaths $\cdot \mathrm{L}^{-1}$, blood pressure $<90 \mathrm{mmHg}$ (systolic) $\leqslant 60 \mathrm{mmHg}$ (diastolic), age $\geqslant 65$ years) was 4 points. Antibiotic treatment with intravenous cefuroxim $1.5 \mathrm{~g}$ three times a day and ciprofloxacine $500 \mathrm{mg}$ twice a day was initiated and methotrexate and CZP were withdrawn. Surprisingly, a chest radiograph showed no pathological findings.

Sputum cultures repeatedly tested negative for bacteria, fungus, influenza and tuberculosis (PCR and microscopy). Quantiferon tests were negative for tuberculosis both before and after CZP.

After 2 weeks of treatment the inflammatory parameters were slowly decreasing but the clinical condition was unchanged. A positron emission tomography-computed tomography (CT) was performed and showed diffuse fluorodeoxyglucose uptake in both lungs, and a low-dose CT examination revealed interstitial changes and ground-glass attenuation. A high-resolution CT (HRCT) scan confirmed these findings. Biopsy and bronchoscopy were not performed due to her clinical condition. A spirometry test showed a restrictive 
TABLE 1 Characteristics of three rheumatoid arthritis patients with certolizumab pegol (CZP)-associated pneumonitis

Case 1

Age years
Sex
Smoking status
Years of rheumatoid arthritis
Rheumatoid factor/anti-CCP
CZP dose
Onset of symptoms
Methotrexate
DAS-28 before CZP
DAS-28 after CZP
HRCT findings
Outcome

Case 2

67
Male
Ex-smoker
34
Positive/NA
NA
15 weeks
None
6.34
6.72
Ground-glass attenuation
Died (18 days after admission)

\author{
71 \\ Female \\ Nonsmoker \\ 8 \\ Positive/positive \\ 8 \\ 16 weeks \\ Yes \\ 5.9 \\ 1.89 \\ Ground-glass attenuation \\ Impaired, home oxygen supply \\ on exertion
}

Case 3

72
Female
Ex-smoker
43
Positive/positive
4
8 weeks
Yes
5.6
5.2
round-glass attenuation
on exertion

CCP: cyclic citrullinated peptide; DAS-28: disease activity score-28 joints; HRCT: high-resolution computed tomography; NA: not answered. \#: number of doses given once in a 2 -week interval; " : time delay between initiation of therapy and onset of symptoms.

pattern with forced expiratory volume in $1 \mathrm{~s}$ (FEV1) $0.86 \mathrm{~L}$ (51\%), forced expiratory volume (FVC) $1.06 \mathrm{~L}$ (52\%) and FEV1/FVC 0.81 . The patient was then started on prednisolone $0.5 \mathrm{mg} \cdot \mathrm{kg}^{-1}$ daily. After 1 month she partly recovered and was discharged with an on-demand oxygen supply due to desaturation on exertion. She never regained her previous lung capacity. A spirometry test showed an FEV1 of $0.67 \mathrm{~L}(40 \%)$ and FVC of $1.27 \mathrm{~L}(62 \%)$ in the outpatient clinic 2.5 months later.

Only two previous cases of CZP-associated pneumonitis have been described. The available information on all three patients is shown in table 1. All three patients were treated with recommended doses of CZP; the two females in combination with methotrexate, the male as monotherapy due to methotrexate-induced pneumonitis 6 years earlier. All showed a clear temporal relationship between the initiation of treatment and the onset of respiratory symptoms. HRCT showed ground-glass attenuation in all three cases. The prognosis is poor. One patient died from respiratory failure 18 days after admission, and the other two patients had impaired lung function, therefore, received home oxygen supply on exertion.

It is well recognised that patients with rheumatoid arthritis frequently develop interstitial lung complications. It is also known that methotrexate can induce interstitial lung disease. In 2006, the Spanish Study Group on Autoimmune Diseases (GEAS) created the Registry bioGEAS in order to collect data on autoimmune diseases secondary to biological therapy (www.biogeas.org). An analysis of 122 cases of interstitial lung disease secondary to different biological agents showed an overwhelming association with TNF- $\alpha$ in $97 \%$ of the 122 cases; rheumatoid arthritis was the underlying disease in $89 \%$. The cases included etanercept, infliximab and adalimumab but not golimumab or CZP. Despite treatment with corticosteroids and withdrawal of the TNF- $\alpha$ inhibitor the prognoses was poor, with only partial resolution in $25 \%$ and an overall mortality of approximately one third [4]. A systematic literature review of non-infectious pulmonary adverse effects to biological treatment was published in 2011, including four cases of pulmonary toxicity associated with golimumab but none to CZP [5]. The three cases presented here show a clear association between CZP treatment and new-onset interstitial lung disease in rheumatoid arthritis patients.

The role of TNF- $\alpha$ inhibitors in the emergence of new-onset interstitial lung disease in patients with rheumatoid arthritis is extremely complex and biased, and the immunopathological mechanism behind this paradox reaction remains unclear. To gain more information on this mechanism, bronchoalveolar lavage and transbronchial biopsies should be performed whenever possible. To assess the prevalence of rare adverse effects, prospective international registries are essential.

Clinicians should be extremely aware of the rare, but potential, risk of acute pneumonitis when treating rheumatoid arthritis patients with CZP and other TNF- $\alpha$ inhibitors. The prognosis is poor and the outcome potentially fatal.

$@$ @RSpublications

When treating rheumatoid arthritis patients with TNF- $\alpha$ inhibitors acute pneumonitis is a rare but potentially fatal risk http://ow.ly/mNcZm 
Jakob Lager, Ole Hilberg, Anders Løkke and Elisabeth Bendstrup

Dept of Pulmonary Medicine, Aarhus University Hospital, Aarhus, Denmark.

Correspondence: J. Lager, Dept of Pulmonary Medicine, Aarhus University Hospital, Nørrebrogade 44, Aarhus, Denmark. E-mail: kabend@rm.dk

Received: April 012013 | Accepted: April 172013

Conflict of interest: Disclosures can be found alongside the online version of this article at www.err.ersjournals.com

Provenance: Submitted article, peer reviewed.

\title{
References
}

Schmitz S, Adams R, Walsh CD, et al. A mixed treatment comparison of the efficacy of anti-TNF agents in rheumatoid arthritis for methotrexate non-responders demonstrates differences between treatments: a Bayesian approach. Ann Rheum Dis 2012; 71: 225-230.

2 Pearce F, Johnson SR, Courtney P. Interstitial lung disease following certolizumab pegol. Rheumatology (Oxford) 2012; 51: 578-580.

Millar A, McKew J, Taggart A. Fatal fibrosing alveolitis with certolizumab. Rheumatology (Oxford) 2012; 51: 953-955. Perez-Alvarez R, Perez-de-Lis M, Diaz-Lagares C, et al. Interstitial lung disease induced or exacerbated by TNFtargeted therapies: analysis of 122 cases. Semin Arthritis Rheum 2011; 41: 256-264.

Hadjinicolaou AV, Nisar MK, Bhagat S, et al. Non-infectious pulmonary complications of newer biological agents for rheumatic diseases - a systematic literature review. Rheumatology (Oxford) 2011; 50: 2297-2305.

\section{Serious bronchopulmonary involvement due to chronic lymphocytic leukaemia}

\author{
To the Editor:
}

Chronic lymphocytic leukaemia (CLL) is the most common type of leukaemia, accounting for $40 \%$ of all cases in individuals $>65$ years of age. Thoracic complications of CLL can be classified into three groups: 1) infectious complications, which are by far the most frequent and are directly related to the severity of immunodeficiency secondary either to leukaemia or therapy; 2) pleural effusions that are either in CLL localisation or related to venous or lymphatic compression caused by a mass or lymph node, and 3) specific bronchopulmonary involvement secondary to lymphocytic infiltration.

Specific bronchopulmonary involvement due to chronic lymphocytic leukaemia (or pathologic leukaemic bronchopulmonary infiltration (LBPI)) is rare and there are few reports in the literature. We report the cases of six patients with CLL and bronchopulmonary leukaemic infiltrates who needed specific treatment due to the severity of respiratory symptoms.

The patients' characteristics are summarised in table S1 in the online supplementary material. All patients gave their written informed consent for inclusion in this report. The six patients were all males. Mean age was 70 years (range 64-74 years). Patient 1 was a current smoker (40 pack-years) and patients 4 and 6 were former smokers (10 PA). CLL had been diagnosed in all patients through a combination of blood hyperlymphocytosis, characteristic lymphocyte morphology and immunophenotyping carried out in the Haematology Department (Tours university Hospital, Tours, France), an average of 6.2 years previously (range 3-14 years). At diagnosis, none of the patients presented with respiratory symptoms or computed tomography (CT) scan abnormalities indicative of LBPI. Patients 1, 2, 4, 5 and 6 had a previous pulmonary history of infectious pneumonia. Only patient 1 had never received specific CLL treatment. All patients were considered to have been in CLL remission for a median of 6 months ( 1 month to 3 years) before the onset of respiratory symptoms.

At the time of onset of respiratory symptoms, four patients were at Binet stage C. Two patients did not have lymphocytosis and could be classified as small lymphocytic lymphoma. All patients had progressive lymphocytosis (four out of six patients) and/or nodal disease (two out of six patients with bilateral axillary and inguinal lymphadenopathies, and all six patients had mediastinal lymphadenopathies). Median lymphocytosis

This article has supplementary material available from err.ersjournals.com 